\title{
Research on the Extendable Data Exchange Standard in Information Integrated Construction of Filling Work
}

\author{
Yoshitaka Minami, Hiroshi Yamamoto, Atsuro Oyama \\ Construction Technology Research Department \\ Public Works Research Institute \\ 1-6, Minamihara, Tsukuba City, Ibaraki Pref., 305-8516, Japan \\ minami@pwri.go.jp, h-yamamo@pwri.go.jp, ooyama55@pwri.go.jp
}

\author{
Toshiyuki Kamei \\ Honshu-Shikoku Bridge Expressway Company Limited \\ 4388-1, Kawazu Town, Sakaide City, Hyogo Pref., \\ 762-0025, Japan \\ toshiyuki-kamei@jb-honshi.co.jp
}

\begin{abstract}
Efforts to promote the information integrated construction of civil engineering work include the study and development of systems for efficiently conducting work and saving labor. However, such systems are often designed for a specific construction site or machinery, so construction information cannot be fully applied to other work sites or construction machinery. It is very important to realize sharing and linking of construction information throughout a CALS (Continuous Acquisition and Life-cycle Support)-based lifecycle, however there is insufficient sharing and linking in the construction stage, especially in the stage before and after the construction. In our research, in order to efficiently complete information integrated construction with sharing and linking of construction information, we proposed an extendible data exchange standard (DES) for filling work, and introduced its review procedure. At last, the requirements of conversion software with efficiently exchanging data by utilizing the DES are summarized.
\end{abstract}

Keywords: Information integrated construction, Information sharing and linking, Information model, Data exchange standard

\section{INTRODUCTION}

Recently, with the development of information technology, computerized information is being effectively used. As computerization develops in the field of civil engineering and construction, much effort is being made on information integrated construction to improve the efficiency of operations by using computerized information. In present information integrated construction involves establishing systems optimized to a specific work site or construction machine in order to improve efficiency and save labor. However, since the information is tailored to a specific purpose, it cannot be fully used for other work sites or construction machineries.

On the other hand, in the Japanese construction industry, one of the major challenges for CALS/EC (Electronic Commerce) is the sharing and linking of information throughout the lifecycle, such as planning, investigation, design, estimation, construction and maintenance. The CALS Action Program 2005 formulated by Japan's Ministry of Land, Infrastructure and Transport (MLIT) in March 2006 calls for a shift in focus from computerizing various information, to sharing and linking information as well as improving processes in order to reduce the product developing time, costs, and to improve productivity. The focus is therefore now on the construction stage, to clearly understand the lack of information sharing and linking with the stages before and after the construction, namely the design and estimation stage and the maintenance stage.

Various data exchange standards have been formulated for information sharing and linking as part of by the construction industry. But since there is no guideline that provides a definitive data exchange standard (DES), it is difficult to apply the information handled by a particular DES to other fields. In addition, if the standards need to be revised, the software that uses those standards must also be modified, which is costly. These problems hinder the spread of DESs.

We are studying a DES to improve work efficiency through the sharing and linking of information in information integrated construction. In our Research on Establishment of a Construction Work Model related to Construction Machinery [1], we discussed modeling of construction information and identified relevant problems. In the Study of Data Exchange for Use by Construction Information Systems [2], the concept of data exchange and a draft of a data model for the DES were proposed. In the Research on building a Data Exchange Standard for Machine Construction [3], we discussed the methods to establish a DES. This technique is linked to ISO 15143 "Earth-moving Machinery and Mobile Road Construction Machinery - Worksite Data Exchange”, currently being discussed for standardization by ISO/TC127/WG2.

\section{OUTLINE OF RESEARCH}

The sharing and linking of information used in various systems for improving operations requires data exchange relatively with considering the meanings of data items used by those systems. The standard must allow data to be exchanged with the meanings' consideration of the data items. In order to enable information to be shared and linked over the entire lifecycle, the DESs formulated in each stage must be linked. This requires a DES developed by the standard procedure. We propose a data exchange 
mechanism using the DES shown in Fig.1 and are conducting Research on building a Data Exchange Standard for Machine Construction [3]. This research suggested that data exchange that considers the meanings of the data items can be realized by using an application schema and data dictionary. The research also introduces the modeling engine.

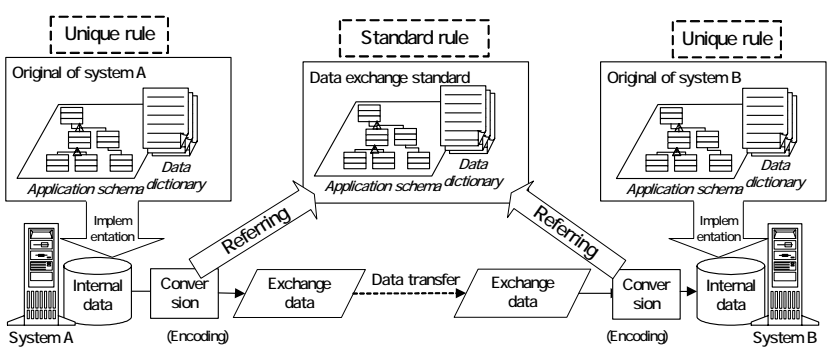

Fig.1 Data exchange using the data exchange standard

Using the results of the previous research, the present research proposes reviewing the DES to enable it to be used for road construction works and summarizes the related requirements. In order to continue using the standard for exchanging data, whenever the standard needs extension or revision because of changes or additions of other factors, the standard must accommodate such changes. We produced a prototype conversion software for general-purpose data exchange and summarized its requirements.

\section{REVIEW PROCEDURE AND REQUIREMENTS OF DATA EXCHANGE STANDARD}

We developed the procedure shown in Fig.2 for a DES that allows data to be exchanged among various systems. The first phase involves investigating existing procedures, data formats and identifying information items to be standardized. The second phase involves preparing application schema and data dictionary proposed in the Research on building a Data Exchange Standard for Machine Construction [3]. The third phase studies a data format that complies with the application schema and data dictionary. The fourth phase involves the construction of prototype conversion software and compatibility verification.

\subsection{Summarization of standardized information items}

One of the requirements of a viable DES is to implement exchanging data among related systems, where it can handle information items used in the existing job sites or systems. This requires a satisfaction of the relevant procedures and an inclusion of the information items of the existing data formats. For the filling work, the relevant procedures include the Fill Compaction Information integrated Construction Management Procedure using TS GPS (proposed) and Nippon Expressway Co., Ltd. Construction Management Procedure (proposed). We investigated the companies registered in the NETIS (New Technology Information System) on MLIT to identify TS
GPS technologies with their necessary information items' summarization for a viable DES.
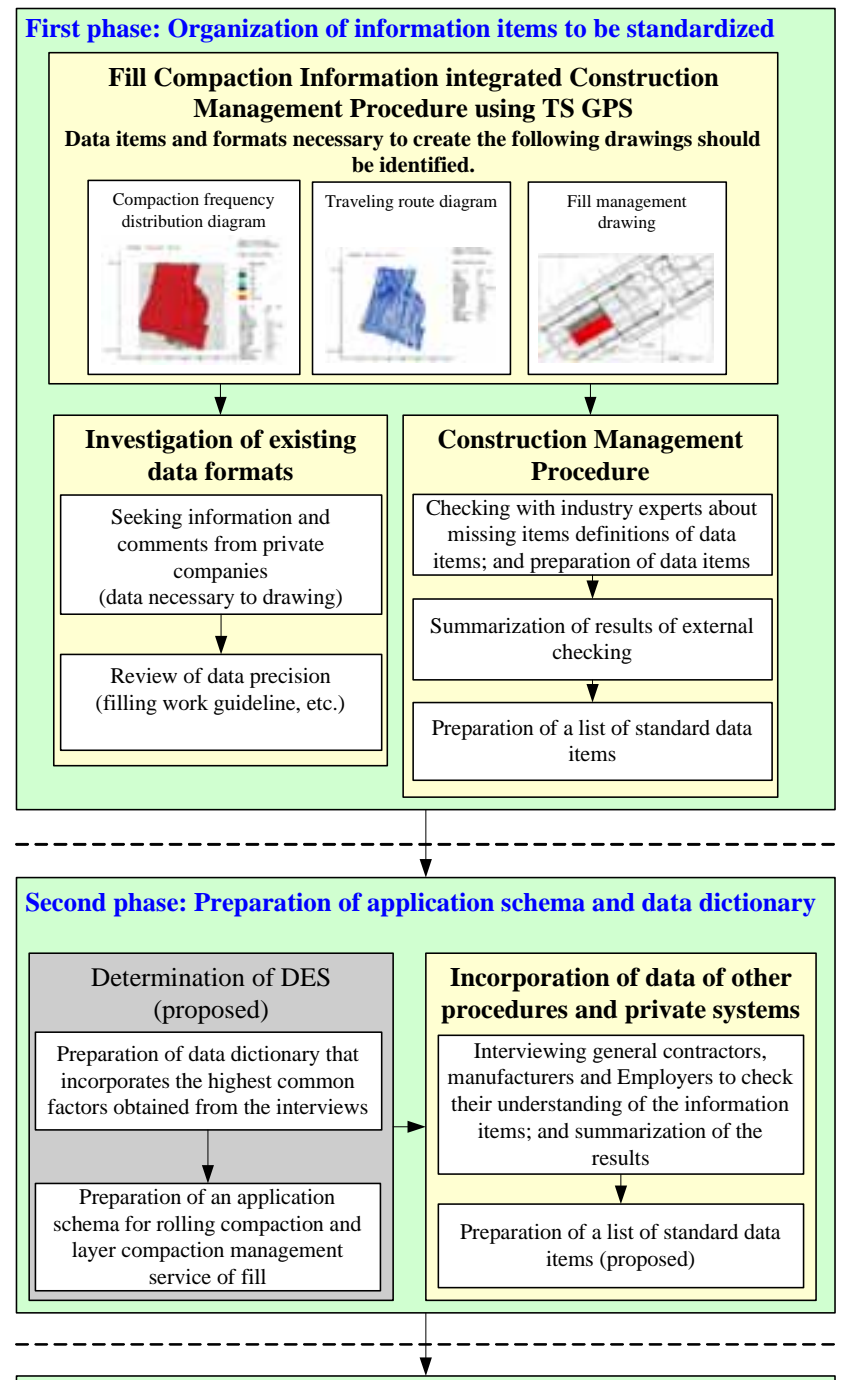

Third phase: Study of proposed data format

Study of the scope of implementation standard proposed for the procedure

Study of file formats (XML, CSV, etc.)

Study of data formats

(in compliance with the structure of the application schema)

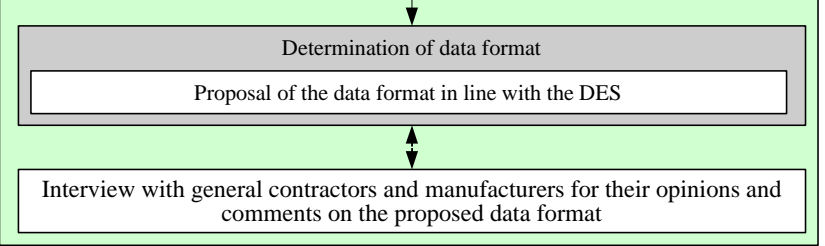

更

Fourth phase: Experimental production of conversion software and verification of compatibility

Defining the functions and requirements of the conversion software

Verification of compatibility

Fig.2 Review procedure of Data Exchange Standard 
The investigation revealed that the information items of the existing DESs can be covered by adding some items to the list in the above Construction Management Procedure. It was also confirmed that information items, item names and notation systems differ among data formats. To ensure data exchange in a viable DES, these differences must be taken into consideration.

\subsection{Application of schema and data dictionary}

When data with different item names and notation systems are exchanged, the DES needs to be able to recognize these data items with the same meanings and to understand the different expression styles. Therefore, the classification structure (or framework), meaning, and expression system of information items, which are the basis of data exchange, should be clearly described. In line with the concept proposed in the Research on building a Data Exchange Standard for Machine Construction [3], the application schema that shows the data's classification structure (or framework) and the data dictionary that shows the meaning and notation system of the data were developed in the proposed DES.

Research on building a Data Exchange Standard for Machine Construction [3] suggested a basic schema. Checking against the results of investigation in the first phase, we found that the relevant classes were indicated. Our proposed application schema is shown in Fig.3.

In addition, the results were organized in terms of compliance with the data dictionary proposed in the Research on building a Data Exchange Standard for Machine Construction [3]. This data dictionary provides strict definitions of data by checking the DataElement table, which gives the meanings of data items, against the ValueDomain table, which gives the data notation. Part of our proposed data dictionary is shown in Table 1.

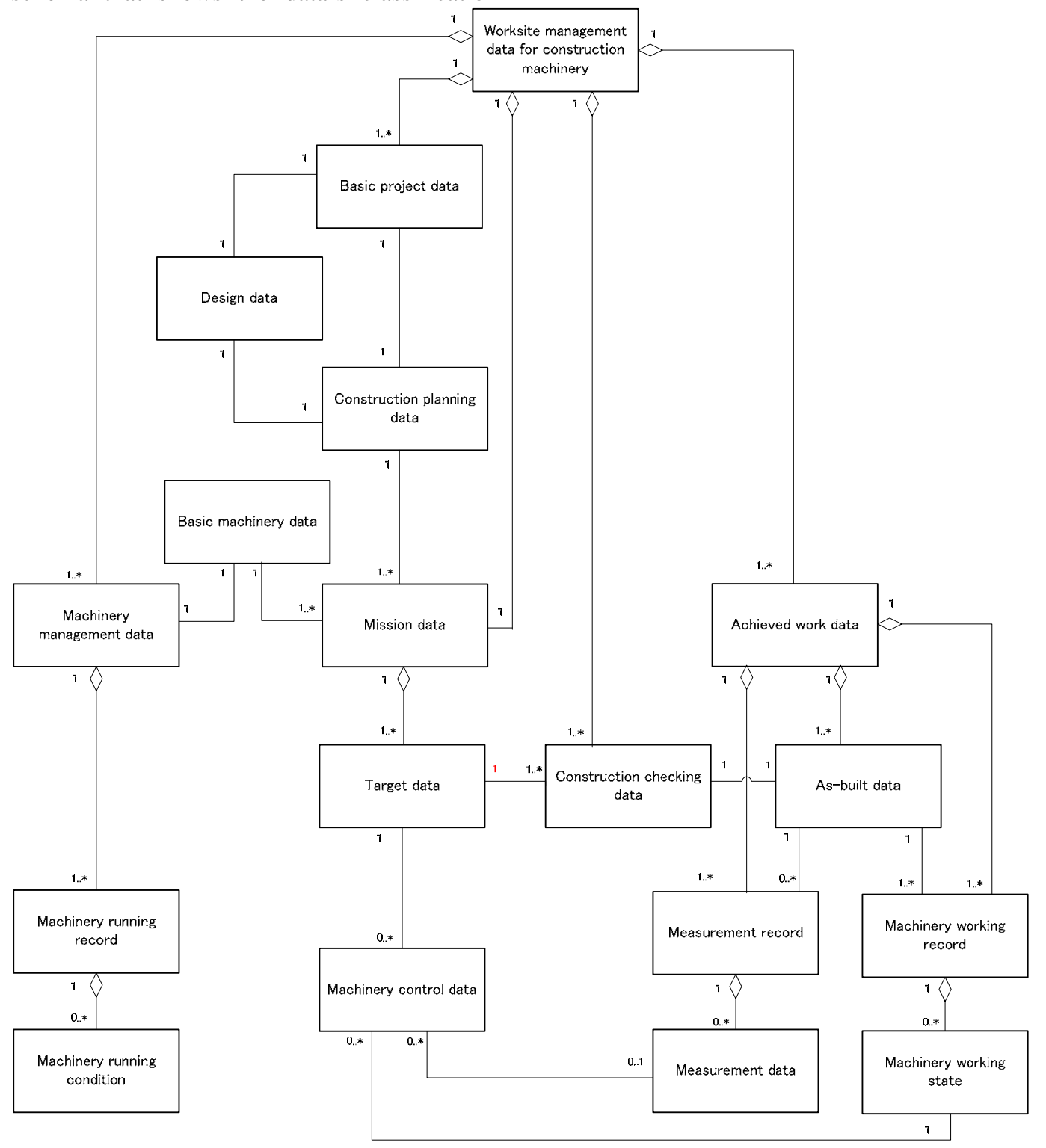

Fig.3 Application schema (proposed) 
Table.1 Part of the proposed data dictionary

\begin{tabular}{|c|c|c|c|c|c|c|}
\hline \multicolumn{4}{|c|}{ Data items } & \multicolumn{3}{|c|}{ Data expressions } \\
\hline No & $\begin{array}{c}\text { Name } \\
\text { ( J apanese) }\end{array}$ & $\begin{array}{c}\text { Name } \\
\text { ( Engl i sh) }\end{array}$ & Definition text & No & $\begin{array}{l}\text { Val ue domai } n \\
\text { name( J apanese) }\end{array}$ & $\begin{array}{l}\text { Val ue domai n } \\
\text { name( Engl i sh) }\end{array}$ \\
\hline A. 01 & 工事名 1 & proj ect_name_1 & $\begin{array}{l}\text { Proj ect name of the } \\
\text { Cont r act. }\end{array}$ & V02- 03 & 工事名 1 & proj ect_name_1 \\
\hline A- 02 & 請負人名 1 & $\begin{array}{l}\text { cont r act or_nam } \\
\text { e_1 }\end{array}$ & $\begin{array}{l}\text { Name of per son or } \\
\text { or gani zat i on that } \\
\text { under takes } \\
\text { const ruct i on work i n } \\
\text { accor dance wi th a } \\
\text { cont r act. }\end{array}$ & V02- 02 & 請負人名 1 & $\begin{array}{l}\text { cont ract or_nam } \\
\text { e_1 }\end{array}$ \\
\hline A- 03 & 工期_自 & $\begin{array}{l}\text { proj ect_begi nn } \\
\text { i ng_date_1 }\end{array}$ & $\begin{array}{l}\text { Begi nni ng date of the } \\
\text { proj ect. }\end{array}$ & V05- 03 & 時間 2 & date_1 \\
\hline A. 04 & 工期_至 & $\begin{array}{l}\text { pr oj ect_compl e } \\
\text { ti on_date_1 }\end{array}$ & $\begin{array}{l}\text { Compl et } i \text { on date of the } \\
\text { proj ect. }\end{array}$ & V05- 03 & 時間 2 & dat e_1 \\
\hline A- 05 & 施工区間_自 & $\begin{array}{l}\text { st at i on_begi nn } \\
\text { i ng_1 }\end{array}$ & $\begin{array}{l}\text { Begi nni ng of st at i on } \\
\text { as ci vi I engi neeri ng } \\
\text { ar ea boundary. }\end{array}$ & V08- 02 & 区間 2 & road_st at i on_2 \\
\hline A- 06 & 施工区間_至 & st at i on_end_1 & $\begin{array}{l}\text { End of st at i on as civi l } \\
\text { engi neer i ng ar ea } \\
\text { boundary. }\end{array}$ & V08 02 & 区間 2 & road_st at i on_2 \\
\hline A- 07 & 平面座標系 & $\begin{array}{l}\text { ref er ence_coor } \\
\text { di nat e_syst em } \\
1\end{array}$ & $\begin{array}{l}\text { Descri bes in fi gur es } \\
\text { the pl anar rect angul ar } \\
\text { coor di nat e syst em of } \\
\text { the work ar ea. }\end{array}$ & V01- 06 & 整数コード 3 & code_i nt eger_3 \\
\hline A- 08 & ローカル使用 & I ocal_fl ag_1 & $\begin{array}{l}\text { Fl ag showi ng whet her } X \\
\text { and } Y \text { coor di nat es in } \\
\text { the measur enent dat a } \\
\text { are I ocal ones or } \\
\text { publ i c ones. If they } \\
\text { are I ocal, ent er } 1 \text {. If } \\
\text { publ i c, ent er } 0 \text {. }\end{array}$ & V01- 02 & 整数コード 1 & code_i nt eger_1 \\
\hline A- 09 & 原点公共座標_X & poi nt_1 & $\begin{array}{l}\text { Descri bes publ i c } \\
\text { coor di nate } X \text { of the } \\
\text { I ocal coor di nate } \\
\text { ori gi } n\end{array}$ & V08 07 & 座標 1 & poi nt_1 \\
\hline A- 10 & 原点公共座標_Y & poi nt_1 & $\begin{array}{l}\text { Descri bes publ i c } \\
\text { coor di nat e Y of the } \\
\text { I ocal coor di nat e } \\
\text { or i gi } n\end{array}$ & V08 07 & 座標 1 & poi nt_1 \\
\hline
\end{tabular}

\subsection{Data format}

A viable DES must have a specific data format to perform data exchange as shown in Fig.1. The optimal format would link with the application schema and data dictionary proposed in the second phase and convey the meanings and expressions. The format must also cope with extension or revision of the DES. Based on this viewpoint, the major existing file formats were compared, and XML was found to be optimal for describing data formats. Fig.4 is a proposed data format defined from the above process.
3.4 Prototype of conversion software and verification of compatibility

The data format proposed in the third phase must be compatible with existing data formats to create a viable DES. Compatibility can be verified by writing experimental conversion software and using it for actual data conversion. For efficient use of existing data, the conversion software must be compatible with varying data formats.

Therefore, in this research we produced data conversion software to identify the requirements for data conversion. 


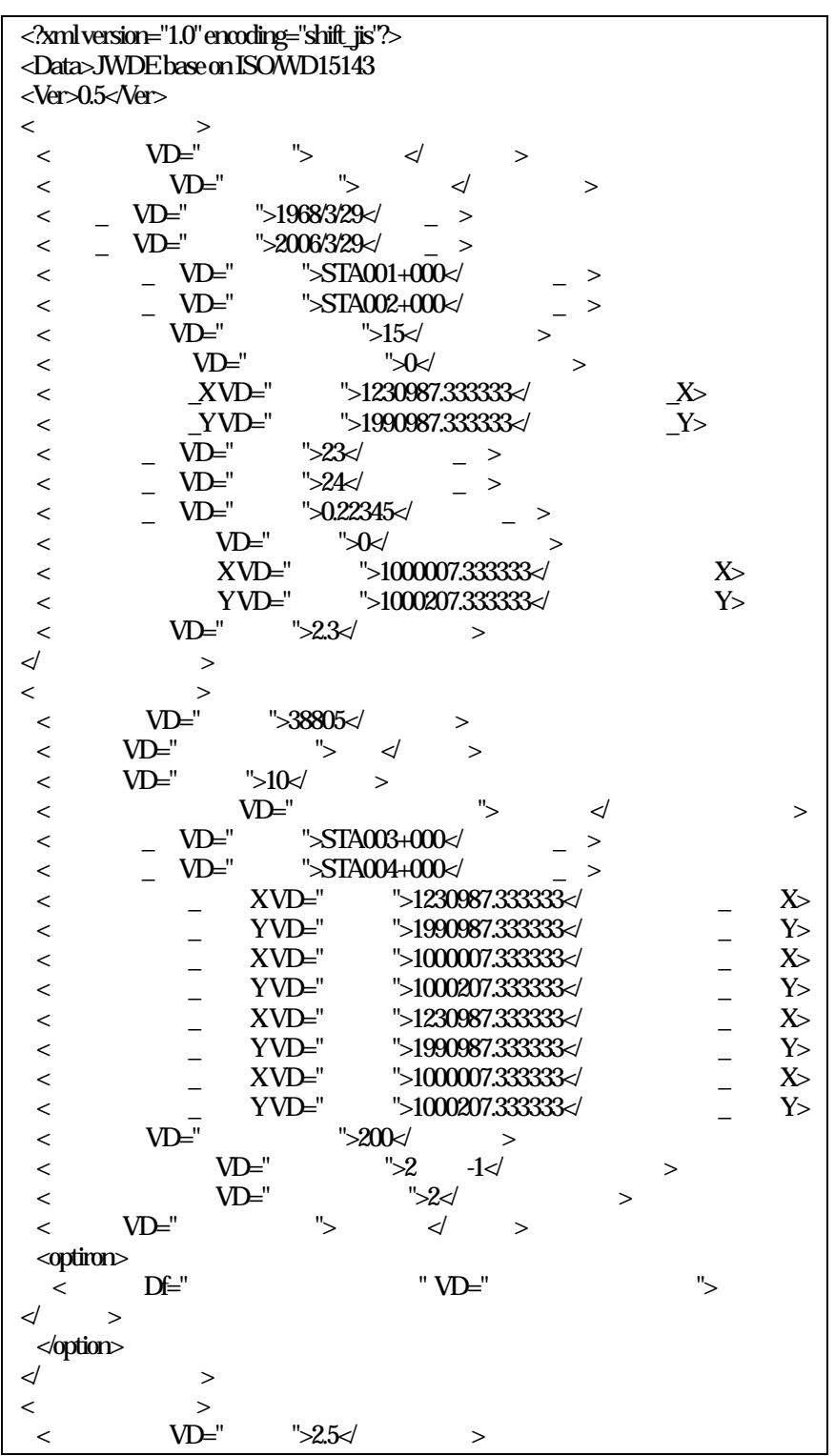

\section{Fig.4 Part of the proposed data format}

\subsubsection{Outline of conversion software}

We intended to develop conversion software that reduces the restrictions imposed by data formats and rarely requires revision. We then developed the software as outlined in Fig.5.

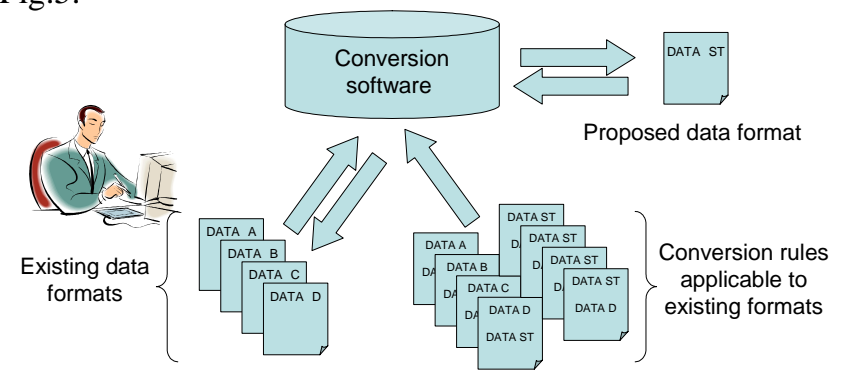

Fig.5 Conversion software

For data conversion by the software, we intended to eliminate the differences among data formats by setting the conversion rules related to data conversion from outside.
With this arrangement, it is not necessary to change the conversion software when dealing with a known data format and thus data exchange can be implemented just by changing the conversion rules. When a data format is unknown, an additional data reading area must be provided to the software to accept the unknown format. Thus, the prototype conversion software will serve as the basic technology for data exchange.

\subsubsection{Operation check result}

An operation check was conducted to confirm compatibility. Two proposed formats, one is JH format and the other is our proposed format, were used for the operation check. The former data format is used for operations by Nippon Expressway and is published in the Construction Management Procedure (proposed). Based on this information, the operation check was made as follows. First, the data of the $\mathrm{JH}$ format was converted to the proposed format. Then, the converted data was reconverted to the original format. And then by setting appropriate operation's data conversion rules any arisen problems during data conversion can be handled.

The results of this operation check confirmed that data was smoothly converted between the $\mathrm{JH}$ format and the proposed format, indicating that the formats are compatible. These findings are used to define the requirements of viable data conversion software.

\subsubsection{Requirements of data conversion software}

The processes of writing experimental data conversion software and checking its operation were done by trial and error. Eventually, it was found that the following requirements must be satisfied to ensure compatibility and flexibility of data conversion.

\section{(1) Order of conversion processing}

The results of the operation check of the experimental conversion software show that the order of conversion plays an important role during the data conversion because a set of data to be converted to another format with effect each other. It is therefore necessary to define the order of conversion to minimize the mutual effect of data items.

\section{(2) Promotion of conversion functions}

The proposed conversion software implemented functions necessary for converting data from the $\mathrm{JH}$ format to the proposed format. It is necessary to develop conversion functions unexpected in the experimental writing of the conversion software in order to improve versatility. Examples include how to handle different data ranges or how to handle numbers used in specific industries.

\section{(3) Promotion of interface}

The proposed conversion software can edit conversion rules, but editing itself has to be done manually. This should be done intuitively and conveniently, while minimizing 
mistakes. Therefore, the appropriate interface should be implemented to reduce the work of converting rules.

(4) Meaning of the data items

In this research, we described the application schema and data dictionary. If conversion rules are written semi-automatically using these results, data will be exchanged more easily and efficiently with the meanings of data items taken into consideration. The required conditions of data conversion will allow the data conversion software to refer to the application schema and data dictionary. The application schema and data dictionary have high compatibility with XML because of their structure and characteristics. Therefore, the data format proposed in our research is considered appropriate.

\section{CONCLUSION}

The authors proposed a data exchange standard for filling work and developed a review procedure. Since the proposed DES has an application schema and data dictionary suggested in the Research on building a Data Exchange Standard for Machine Construction [3], it is possible to extend the DES to handle additional trades or data exchanges to a specific construction site. We also implemented data conversion software and demonstrated what is necessary to make sure the software flexibility to efficiently realize data exchange with varying data formats. If data exchange software is developed based on those requirements, the software would allow the sharing and linking of information among various systems.

Our research provides an efficient method for successful completing sharing and linking of data information in construction industry and other applications. It contributes to a prompt spread of the DES and improves the efficiency of related business.

\section{ACKNOWLEDGMENT}

The authors would like to thank ISO/TC127/WG2, the Ministry of Land, Infrastructure and Transport, its Regional Bureaus, Japan Construction Mechanization Association, and Nippon Koei Co., Ltd. for their supporting in our research.

\section{REFERENCES}

[1] Hirofumi Hirashita, Tadashi Yoshida: Review on Construction of a Model for Construction Information related to Construction Machinery, Summary of Lectures for Technical Research Presentations of the Kanto Branch of the Japan Society of Civil Engineers, Kanto Branch of the Japan Society of Civil Engineers, 2003.3.

[2] Tadashi Yoshida, Hirofumi Hirashita: Study of Data Exchange for Use by Construction Information Systems, 20th International Symposium on Automation and Robotics in Construction, The International Association for Automation and Robotics in Construction (ISARC), pp.611-616, 2003.9.

[3] Atsuro Oyama, Hiroshi Yamamoto, Masayasu Nihei, Yoshitaka Minami : Research on building a Data Exchange Standard for Machine Construction, Journal of Applied Computing in Civil Engineering, Vol.15, 2006.10(being submitted) 